\title{
Some Inequalities for Generalized Commutators
}

\author{
By
}

\author{
Khristo N. BoYADZHIEV*
}

\begin{abstract}
Let $A, B$ be linear operators on a Banach space $\mathscr{H}$ with spectra in the set $S=\mathbf{C} \backslash(-\infty, 0)$, for which
\end{abstract}

$$
\left\|t(A+t)^{-1}\right\| \leqq M,\left\|t(B+t)^{-1}\right\| \leqq N \quad(t>0) .
$$

Then for a certain class of holomorphic functions $f$ preserving $S$ and $f(0)=\lim _{s \rightarrow 0+} f(s)=0$, one has

$$
\|f(A) X-X f(B)\| \leqq a f(b\|A X-X B\|) \quad \text { for all } X \in B(\mathscr{H}),\|X\| \leqq 1,
$$

where $a=2(M+N), b=M N /(M+N)$.

At that, if $\alpha \in \mathbf{C}, 0<\operatorname{Re} \alpha \leqq 1$, then for all $X \in B(\mathscr{H})$

$$
\left\|A^{\alpha} X-X B^{\alpha}\right\| \leqq C(M, N, \alpha)\|X\|^{1-\operatorname{Re} \alpha}\|A X-X B\|^{\operatorname{Re} \alpha}
$$

where

$$
C(M, N, \alpha)=\frac{|\sin \alpha \pi|(M N)^{\operatorname{Re} \alpha}(M+N)^{1-\operatorname{Re} \alpha}}{\pi \operatorname{Re} \alpha(1-\operatorname{Re} \alpha)} .
$$

Also

$$
\left\|\exp \left(-t A^{1 / 2}\right) X-X \exp \left(-t B^{1 / 2}\right)\right\| \leqq C(M, N) t^{2 / 3}\|X\|^{2 / 3}\|A X-X B\|^{1 / 3},
$$

where

$$
C(M, N)=3 \pi^{-1}(M N)^{1 / 3}(M+N)^{2 / 3}, t \geqq 0, X \in B(\mathscr{H}) .
$$

\section{§1. Introduction}

Recently F. Kittaneh and H.Kosaki [4] obtained the interesting inequalities:

Let $A, B$ be two positive operators on a Hilbert space $\mathscr{H}$ and $f$-an operator monotone function on $(0, \infty)$. Then

$$
\begin{aligned}
& \text { If } \lim _{s \rightarrow++} f(s)=0, \text { one has } \\
& \|f(A)-f(B)\| \leqq f(\|A-B\|),
\end{aligned}
$$

Communicated by H. Araki, August 8, 1989.

* Institute of Mathematics, Bulgarian Acad. Sci., 1090 Sofia, P.O.Box 373, Bulgaria

Current address: Department of Mathematics, Ohio Northern University, Ada, Ohio 45810, U.S.A. 


$$
\begin{aligned}
& \text { If } A \geqq a \geqq 0 \text { and } B \geqq b \geqq 0 \text {, one has for all } X \in B(\mathscr{H}) \\
& \|f(A) X-X f(B)\|_{p} \leqq C(a, b)\|A X-X B\|_{p}, 1 \leqq p \leqq \infty,
\end{aligned}
$$

where

$$
C(a, b)=\left\{\begin{array}{ll}
\frac{f(a)-f(b)}{a-b} & a \neq b \\
f^{\prime}(a) & a=b
\end{array} .\right.
$$

In particular, when $f(s)=s^{\alpha}, 0<\alpha \leqq 1$, and $A \geqq c>0, B \geqq c>0$, (1.2) turns into

$$
\left\|A^{\alpha} X-X B^{\alpha}\right\|_{p} \leqq \alpha c^{\alpha-1}\|A X-X B\|_{p} \quad(X \in B(\mathscr{H})) .
$$

Unfortunately, when $c=0$, or $a=b=0$ and $f^{\prime}(a)=\infty$ in (1.2), it is impossible to estimate $\|f(A) X-X f(B)\|_{p}$ in terms of $\|A X-X B\|_{p}$ there even for $p=\infty$.

Such an estimate is sometimes important. For instance, in another development-for use in $C^{*}$-algebra theory, W. Arveson proved the following result (see [1], the Lemma on p. 332)

(1.4) Let $\mathscr{U}$ be a $C^{*}$-algebra, $f$-a continuous function on $[0,1]$ (if $\mathscr{U}$ has no unit, one assumes $f(0)=0$ ) and let $\varepsilon>0$. There exists $\delta>0$ such that any time when $a, x$ are in the unit ball of $\mathscr{U}$ and $a \geqq 0$, one has

$$
\|a x-x a\|<\delta \quad \text { implies }\|f(a) x-x f(a)\|<\varepsilon .
$$

For $f(s)=s^{\alpha}, 0<\alpha<1$, a more precise estimate was found in the paper [7] (Lemma 2.1):

(1.5) If $a, x$ are elements in a $C^{*}$-algebra and $a \geqq 0$, then for any $\alpha, 0<\alpha<1$ one has

$$
\left\|a^{\alpha} x-x a^{\alpha}\right\| \leqq(1-\alpha)^{\alpha-1}\|x\|^{1-\alpha}\|a x-x a\|^{\alpha} .
$$

As mentioned in the remarks on p. 4 of [7], U. Haagerup has reduced the constant $(1-\alpha)^{\alpha-1}$ in $(1.5)$ to $\sin \alpha \pi(\pi \alpha(1-\alpha))^{-1}$. We have come to this result independently and we present the refined inequality here in a general setting-see below (2.6).

The aim of these notes is to describe a method of obtaining inequalities for generalized commutators and to illustrate it by some examples, thus complementing the results of Kittaneh-Kosaki. The inequalities here are stated for Banach space operators, although Banach algebra elements could be used also. The operator framework keeps in line with the notations in KittanehKosaki's paper and makes it possible to consider unbounded operators as well. 


\section{§2. Inequalities}

We consider operator monotone functions $f$ on $[0, \infty)$ of the form

$$
\begin{aligned}
& f(z)=k z+\int_{0}^{\infty} \frac{z}{z+t} d \mu(t), \quad k \geqq 0, \\
& f(0)=\lim _{s \rightarrow 0+} f(s)=0, \int_{1}^{\infty} d \mu(t) / t<\infty, \quad \mu(0)=0,
\end{aligned}
$$

and for the monotone increasing function $\mu$ we assume also that $\mu^{\prime}$ exists and is positive in $(0, \infty)$. We also consider a pair of two bounded linear operators $A, B$ on a complex Banach space $\mathscr{H}$ satisfying

$$
\begin{aligned}
& \operatorname{Sp}(A), \operatorname{Sp}(B) \subset \mathbf{C} \backslash(-\infty, 0), \\
& \left\|t(A+t)^{-1}\right\| \leqq M,\left\|t(B+t)^{-1}\right\| \leqq N \quad \text { for all } t>0 .
\end{aligned}
$$

For such functions $f$ and operators $A, B$ one can define

$$
f(A)=k A+\int_{0}^{\infty} A(A+t)^{-1} d \mu(t) \quad \text { and similarly } f(B) .
$$

As $\quad \int_{1}^{\infty}\left\|A(A+t)^{-1}\right\| d \mu(t) \leqq M\|A\| \int_{1}^{\infty} \frac{d \mu(t)}{t}<\infty$

and

$$
\int_{0}^{1}\left\|A(A+t)^{-1}\right\| d \mu(t)=\int_{0}^{1}\left\|1-t(A+t)^{-1}\right\| d \mu(t) \leqq(M+1) \int_{0}^{1} d \mu(t)<\infty,
$$

the above integral is a uniformly convergent Bochner-Stieltjes operator valued integral and $f(A)$ is a bounded linear operator on $\mathscr{H}$.

Let $X$ be in the unit ball of $B(\mathscr{H})$ and put $c=\|A X-X B\|$. For all $s \geqq 0$ we have

$$
\begin{aligned}
& \|f(A) X-X f(B)\|=\| k(A X-X B)+\int_{0}^{s} t\left(X(B+t)^{-1}-(A+t)^{-1} X\right) d \mu(t) \\
& +\int_{s}^{\infty} t(A+t)^{-1}(A X-X B)(B+t)^{-1} d \mu(t) \| \\
& \quad \leqq k c+(M+N) \mu(s)+c M N \int_{s}^{\infty} \frac{d \mu(t)}{t} .
\end{aligned}
$$

This last expression takes its minimum for $s \geqq 0$ in $s=\lambda=c M N /(M+N)$, as its derivative with respect to $s$ for $s>0$ is

$$
\mu^{\prime}(s)\left(M+N-c M N s^{-1}\right) .
$$

Let $a=2(M+N)$ and let $M N \geqq 1 / 2$ or $k=0$. One easily checks that 


$$
\begin{aligned}
k c+ & (M+N) \int_{0}^{\lambda} d \mu(t)+c M N \int_{\lambda}^{\infty} d \mu(t) / t \\
& \leqq a\left(k \lambda+\int_{0}^{\lambda} \frac{\lambda}{\lambda+t} d \mu(t)+\int_{\lambda}^{\infty} \frac{\lambda}{t+\lambda} d \mu(t)\right)=a f(\lambda) .
\end{aligned}
$$

This way we have proved the theorem:

Theorem 2.1. Let $A, B$ be two operators on the Banach space $\mathscr{H}$ satisfying (2.2). Then for every function $f$ of the form (2.1) and every $X \in B(\mathscr{H}),\|X\| \leqq 1$ :

$$
\|f(A) X-X f(B)\| \leqq a f(b\|A X-X B\|)
$$

where $a=2(M+N), b=M N /(M+N)$ and $M N \geqq 1 / 2$ or $k=0$.

Note that when $M N \geqq 1$ and

$$
\int_{0}^{\infty} d \mu(t) / t<\infty \text {, i.e. } f^{\prime}(0) \text { is finite, it follows from (2.3) for } s=0 \text { : }
$$

$$
\|f(A) X-X f(B)\| \leqq f^{\prime}(0) M N\|A X-X B\| \quad \text { for all } X \in B(\mathscr{H})
$$

in accordance with (1.2).

We shall present now one variety of (2.4) which is of particular interest.

Starting from the representation

$$
z^{\alpha}=\frac{\sin \alpha \pi}{\pi} \int_{0}^{\infty} \frac{z}{z+t} t^{\alpha-1} d t, z \in \mathbb{C} \backslash(-\infty, 0), 0<\operatorname{Re} \alpha \leqq 1
$$

one can define the fractional powers

$$
A^{\alpha}=\frac{\sin \alpha \pi}{\pi} \int_{0}^{\infty}(A+t)^{-1} A t^{\alpha-1} d t \text { and in the same way } B^{\alpha} \text { (see [2]). }
$$

Proposition 2.2. For $A, B$ satisfying (2.2), $0<\operatorname{Re} \alpha \leqq 1$, and all $X \in B(\mathscr{H})$ one has

$$
\left\|A^{\alpha} X-X B^{\alpha}\right\| \leqq C(M, N, \alpha)\|X\|^{1-\operatorname{Re} \alpha}\|A X-X B\|^{\operatorname{Re} \alpha}
$$

where $\quad C(M, N, \alpha)=\frac{|\sin \alpha \pi|}{\pi \operatorname{Re} \alpha(1-\operatorname{Re} \alpha)}(M N)^{\operatorname{Re} \alpha}(M+N)^{1-\operatorname{Re} \alpha}$

Proof. Proceeding as in (2.3) we find for every $s>0$ and $X \in B(\mathscr{H})$

$$
\begin{aligned}
& \left\|A^{\alpha} X-X B^{\alpha}\right\| \\
& \quad \leqq \frac{|\sin \alpha \pi|}{\pi}\left(\|X\|(M+N) \int_{0}^{s}\left|t^{\alpha-1}\right| d t+\|A X-X B\| M N \int_{s}^{\infty}\left|t^{\alpha-2}\right| d t\right)
\end{aligned}
$$




$$
\leqq \frac{|\sin \alpha \pi|}{\pi}\left(\frac{\|X\|(M+N)}{\operatorname{Re} \alpha} s^{\operatorname{Re} \alpha}+\frac{\|A X-X B\| M N}{1-\operatorname{Re} \alpha} s^{\operatorname{Re} \alpha-1}\right) \quad\left(\text { as }\left|t^{\gamma}\right| \leqq t^{\operatorname{Re} \gamma}\right) .
$$

Minimizing the right hand side for $s>0$ we get (2.6).

Proposition 2.3. Let $H$ be a Hilbert space, $\|\cdot\|_{p}$-the Schatten norm, $1 \leqq p$ $\leqq \infty, f-a$ function as in (2.1) and $A, B \in B(\mathscr{H})$-operators satisfying (2.2). Then one has

$$
\|f(A) X-X f(B)\|_{p} \leqq a f\left(b\|A X-X B\|_{p}\right) \quad \text { for all } X \in B(\mathscr{H}),\|X\|_{p} \leqq 1,
$$

where $a=2(M+N), b=M N /(M+N)$ and either $M N \geqq 1 / 2$ or $k=0$.

Also

$$
\left\|A^{\alpha} X-X B^{\alpha}\right\|_{p} \leqq C(M, N, \alpha)\|X\|_{p}^{1-\operatorname{Re} \alpha}\|A X-X B\|_{p}^{\operatorname{Re} \alpha}
$$

for all $X \in B(\mathscr{H}),\|X\|_{p}<\infty, 0<\operatorname{Re} \alpha \leqq 1$ and $C(M, N, \alpha)$ as in (2.6).

Proof. We repeat the proofs of (2.4) and (2.6), using $\|\cdot\|_{p}$ instead of $\|\cdot\|$ and estimating on the right hand sides of the inequalities in the following way:

$$
\begin{aligned}
& \left\|X(B+t)^{-1}-(A+t)^{-1} X\right\|_{p} \leqq(M+N)\|X\|_{p} t^{-1}, \\
& \left\|(A+t)^{-1}(A X-X B)(B+t)^{-1}\right\|_{p} \leqq M N\|A X-X B\|_{p} t^{-2} .
\end{aligned}
$$

Remarks. The technique used in the above proofs-dividing the integral in two parts on $[0, s]$ and $[s, \infty)$ estimated in different ways and then minimizing for $s>0$-is not new. It has been used, for instance, by Matsaev and Palant [6] for obtaining the inequality

$$
\begin{aligned}
& \left\|A^{\alpha}-B^{\alpha}\right\| \leqq C(M, N, \alpha)\|A-B\|^{\alpha} \\
& (0<\alpha \leqq 1, C(M, N, \alpha) \text { as in (2.6)) }
\end{aligned}
$$

for operators $A, B$ essentially as in (2.2).

This method has been used also for proving moment type inequalities

$$
\begin{aligned}
& \left\|A_{x}^{\alpha}\right\| \leqq C(M, \alpha)\|x\|^{1-\alpha}\|A x\|^{\alpha} \quad(x \in \mathscr{H}) \\
& (0<\alpha \leqq 1, C(M, \alpha)=C(M, 1, \alpha))
\end{aligned}
$$

for $A$ as in (2.2)-see [5], [8].

Note that Matsaev-Palant's inequality (2.9) follows immediately from (2.6) by putting there $X=1=$ identity operator. In the same way we obtain from (2.4) the immediate corollary:

Corollary 2.4. Let $f$ be as in (2.1) and let A, B satisfy (2.2). Then

$$
\|f(A)-f(B)\| \leqq a f(b\|A-B\|)
$$


( $a, b$ as in (2.4)), which is a Banach space variety of (1.1).

In this connection see also Theorem 3.4 in [4]. A natural modification of the proof turns it into a theorem for generalized commutators.

\section{§3. Further Inequalities}

We want to point out that many functions of the form

$$
f(z)=\int_{0}^{\infty} \frac{z}{z+t} g(t) d t, \text { or } f(z)=\int_{0}^{\infty} \frac{h(t)}{z+t} d t, z \in \mathbb{C} \backslash(-\infty, 0),
$$

with explicitly given $g, h$ can successfully be used for obtaining moment type inequalities for generalized commutators via the above method. To illustrate this we shall present the following example:

$$
\exp \left(-t z^{1 / 2}\right)=\frac{1}{\pi} \int_{0}^{\infty} \frac{\sin (t \sqrt{\lambda})}{z+\lambda} d \lambda \quad(t \geqq 0)(\text { see }[2],[10]) .
$$

It is well-known that if $A$ is a closed linear operator satisfying (2.2), its square root $A^{1 / 2}$ (defined as in Section 2) is the generator of a (holomorphic) oneparameter operator semigroup which can be determined by the formula

$$
\exp \left(-t A^{1 / 2}\right)=\frac{1}{\pi} \int_{0}^{\infty}(A+\lambda)^{-1} \sin (t \sqrt{\lambda}) d \lambda, \quad t \geqq 0,
$$

(see [2], Section 5).

Proposition 3.1. Let $A, B$ be closed (possibly unbounded) linear operators on the Banach space $\mathscr{H}$ satisfying (2.2). Then for any $X \in B(\mathscr{H})$ for which $A X$ $-X B$ is bounded, we have

$$
\begin{aligned}
& \left\|\exp \left(-t A^{1 / 2}\right) X-X \exp \left(-t B^{1 / 2}\right)\right\| \leqq C(M, N) t^{2 / 3}\|X\|^{2 / 3}\|A X-X B\|^{1 / 3}, \\
& C(M, N)=3 \pi^{-1}(M N)^{1 / 3}(M+N)^{2 / 3}, \quad t \geqq 0 .
\end{aligned}
$$

Proof. For all $s>0$ :

$$
\begin{aligned}
& \pi\left\|\exp \left(-t A^{1 / 2}\right) X-X \exp \left(-t B^{1 / 2}\right)\right\| \\
& \leqq t \int_{0}^{s}\left|\frac{\sin (t \sqrt{\lambda})}{t \sqrt{\lambda}}\right| \sqrt{\lambda}\left\|(A+\lambda)^{-1} X-X(B+\lambda)^{-1}\right\| d \lambda \\
& \quad+\int_{s}^{\infty}|\sin (t \sqrt{\lambda})|\left\|(A+\lambda)^{-1}(A X-X B)(B+\lambda)^{-1}\right\| d \lambda \\
& \leqq 2 t\|X\|(M+N) \sqrt{s}+M N\|A X-X B\| s^{-1} .
\end{aligned}
$$

Minimizing this for $s>0$ we come to (3.2). 


\section{References}

[1] Arveson, W., Notes on extensions of $C^{*}$-algebras, Duke Math. J., 44(1977), 329-355.

[2] Balakrishnan, A.V., Fractional powers of closed operators and the semigroups generated by them, Pacific J. Math., 10(1960), 419-437.

[3] Hennings, M.A., Kasparov's technical lemma for $b^{*}$-algebras, Math. Proc. Cambridge Philos. Soc., 105(1989), 537-545.

[4] Kittaneh, F. and Kosaki, H., Inequalities for the Schatten p-norm V, Publ. RIMS, Kyoto Univ., 23(1986), 433-443.

[5] Krein, S.G., Linear Differential Equations in Banach Spaces, Translations Amer. Math. Soc., 29, Providence, RI (1971).

[6] Matsaev, V.I. and Palant, Yu.A., On the powers of a bounded dissipative operator, Ukrain. Mat. Zh., 14(1962), 329-337 (in Russian).

[7] Olsen, C.L. and Pedersen, G.K., Corona $C^{*}$-algebras and their applications to lifting problems, Preprint.

[8] Yosida, K., A perturbation theorem for semi-groups of linear operators, Proc. Japan Acad. Ser. A Math. Sci., 41(1965), 645-647.

[9] - Functional Analysis, (6th edition) Springer Verlag, Berlin, 1980.

[10] Widder, D.V., A transform related to the Poisson integral for a half-plane, Duke Math. J., 33(1966), 355-362. 
